\title{
Some generalized Hermite-Hadamard-Fejér inequality for convex functions
}

\author{
Miguel Vivas-Cortez ${ }^{*}$ (D), Péter Kórus² and Juan E. Nápoles Valdés ${ }^{3,4}$
}

\author{
"Correspondence: \\ mjvivas@puce.edu.ec \\ ${ }^{1}$ Faculty of Exact and Natural \\ Sciences, School of Physical \\ Sciences and Mathematics, \\ Pontifical Catholic University of \\ Ecuador, Av. 12 de octubre 1076 y \\ Roca, Apartado Postal 17-01-2184, \\ Sede Quito, Ecuador \\ Full list of author information is \\ available at the end of the article
}

\begin{abstract}
In this paper, we have established some generalized inequalities of Hermite-Hadamard-Fejér type for generalized integrals. The results obtained are applied for fractional integrals of various type and therefore contain some previous results reported in the literature.
\end{abstract}

MSC: $26 \mathrm{~A} 33 ; 26 \mathrm{~A} 51 ; 26 \mathrm{D} 10$

Keywords: Integral inequalities; Fractional integrals; Convex functions

\section{Preliminaries}

Consider a certain function $f:[a, b] \subseteq \mathbb{R} \rightarrow \mathbb{R}$. This function is said to be convex if

$$
f(t x+(1-t) y) \leq t f(x)+(1-t) f(y)
$$

is satisfied for any $x, y \in[a, b]$, being $t \in[0,1]$. Likewise, $f$ is called concave if $-f$ is convex.

One of the most important inequalities that have attracted many experts in the last few decades is the famous Hermite-Hadamard inequality

$$
f\left(\frac{a+b}{2}\right) \leq \frac{1}{b-a} \int_{a}^{b} f(x) d x \leq \frac{f(a)+f(b)}{2}
$$

that holds for any function $f$ convex on the interval $[a, b]$. This inequality was published by Hermite in 1883 and, independently, by Hadamard in 1893. It gives an estimation of the mean value of a convex function, and it is important to note that it also provides a refinement to the Jensen inequality. The interested reader is referred to $[3,4,10,13-16$, $21,22,27-30]$ and the references therein for more information and other extensions of the Hermite-Hadamard inequality.

In this paper, following that path, we present a new integral operator (that we could call "integral of one function with respect to another" for reasons that we will see later) and apply it to the study of generalized inequalities, more general than (1.1), this time the so-called Hermite-Hadamard-Fejér inequality.

We assume that the reader is familiar with the classic definition of the Riemann integral, so we do not present it.

(c) The Author(s) 2021. This article is licensed under a Creative Commons Attribution 4.0 International License, which permits use, sharing, adaptation, distribution and reproduction in any medium or format, as long as you give appropriate credit to the original author(s) and the source, provide a link to the Creative Commons licence, and indicate if changes were made. The images or other third party material in this article are included in the article's Creative Commons licence, unless indicated otherwise in a credit line to the material. If material is not included in the article's Creative Commons licence and your intended use is not permitted by statutory regulation or exceeds the permitted use, you will need to obtain permission directly from the copyright holder. To view a copy of this licence, visit http://creativecommons.org/licenses/by/4.0/. 
As we know, basically there are two types of fractional differential operators, RiemannLiouville and Caputo, respectively:

$$
\begin{aligned}
& { }^{R L} D^{\alpha} f(t)=\frac{d}{d t}\left\{J_{F, a}^{1-\alpha}(f)(t)\right\}, \\
& { }^{C} D^{\alpha} f(t)=J_{F, a}^{1-\alpha}\left(\frac{d f}{d t}\right)(t),
\end{aligned}
$$

with certain kernel $F$. In particular, we will deal with real integral operators defined on $\mathbb{R}$ of the RL-type.

One of the first operators that can be called fractional is the Riemann-Liouville fractional derivative of order $\alpha \in \mathbb{C}, \operatorname{Re}(\alpha) \geq 0$ defined by [6].

Definition 1.1 Let $f \in L^{1}((a, b) ; \mathbb{R}),(a, b) \in \mathbb{R}^{2}, a<b$. The right- and left-hand side Riemann-Liouville fractional integrals of order $\alpha>0$ are defined by

$$
\begin{aligned}
& { }^{R L} J_{a^{f}}^{\alpha} f(t)=\frac{1}{\Gamma(\alpha)} \int_{a}^{t}(t-s)^{\alpha-1} f(s) d s, \quad t>a, \\
& { }^{R L} J_{b^{-}}^{\alpha} f(t)=\frac{1}{\Gamma(\alpha)} \int_{t}^{b}(s-t)^{\alpha-1} f(s) d s, \quad t<b,
\end{aligned}
$$

and their corresponding differential operators are given by

$$
\begin{aligned}
& D_{a^{+}}^{\alpha} f(t)=\frac{d}{d t}\left({ }^{R L} J_{a^{+}}^{1-\alpha} f(t)\right)=\frac{1}{\Gamma(1-\alpha)} \frac{d}{d t} \int_{a}^{t} \frac{f(t)}{(t-s)^{\alpha}} d s, \\
& D_{b^{-}}^{\alpha} f(t)=-\frac{d}{d t}\left({ }^{R L} J_{b^{-}}^{1-\alpha} f(t)\right)=-\frac{1}{\Gamma(1-\alpha)} \frac{d}{d t} \int_{t}^{b} \frac{f(t)}{(s-t)^{\alpha}} d s .
\end{aligned}
$$

There have been many fractional integrals defined and investigated in the recent years, for example, so-called Hadamard, Katugampola, $k$-fractional integrals, etc. (see e.g. [1, 2, $11,17,23,24,26])$.

Now, we give the definition of a general fractional integral. Throughout the work we consider that the integral operator kernel $T$ defined below is an absolutely continuous function (see [8]).

Definition 1.2 Let $I$ be an interval $I \subseteq \mathbb{R}, a, b, t \in I$, and $\alpha \in \mathbb{R}$. The integral operators $J_{T}^{\alpha}$, right and left, are defined for every locally integrable function $f$ on $I$ as follows:

$$
\begin{array}{ll}
J_{T, a_{+}}^{\alpha}(f)(t) & =\int_{a}^{t} \frac{f(s)}{T(t-s, \alpha)} d s, \quad t>a, \\
J_{T, b_{-}}^{\alpha}(f)(t) & =\int_{t}^{b} \frac{f(s)}{T(s-t, \alpha)} d s, \quad b>t .
\end{array}
$$

It is easy to see that the case of the $J_{T}^{\alpha}$ operators defined above contains, as particular cases, the integral operators obtained from conformable and non-conformable local derivatives. However, we will see that it goes much further by containing the cases presented before. It is clear that from our definition new extensions and generalizations of known integral operators can be defined and many integral operators can be considered 
particular cases of the integral operator defined above, we let the reader complete this analysis. For simplicity of notation, let us denote (see [14])

$$
\begin{aligned}
& (a, b) J_{T, a+}^{\alpha}(f)(b)=\int_{a}^{b} \frac{f(s)}{T\left(\frac{b-s}{b-a}, \alpha\right)} d s, \\
& (a, b) J_{T, b-}^{\alpha}(f)(a)=\int_{a}^{b} \frac{f(s)}{T\left(\frac{s-a}{b-a}, \alpha\right)} d s .
\end{aligned}
$$

We also use the "central" integral operator defined by (see [31])

$$
J_{T, a}^{\alpha}(f)(b)=\int_{a}^{b} \frac{f(t)}{T(t, \alpha)} d t, \quad b>a .
$$

We present the definition of generalized derivative that was defined in [19].

Definition 1.3 For a function $f:[0, \infty) \rightarrow \mathbb{R}$, the $N_{T}^{\alpha}$-derivative of $f$ is defined by

$$
N_{T}^{\alpha} f(t)=\lim _{\varepsilon \rightarrow 0} \frac{f(t+\varepsilon T(t, \alpha))-f(t)}{\varepsilon}
$$

for all $t>0, \alpha \in(0,1), T(t, \alpha)$ being some function.

We can also define the lateral derivative operators (right and left) in the case of our generalized derivative. For this, it is sufficient to consider them from the corresponding integral operator. To do this, just make use of the fact that if $f$ is differentiable, then $N_{T}^{\alpha} f(t)=T(t, \alpha) f^{\prime}(t)$, where $f^{\prime}(t)$ is the ordinary derivative. For the right derivative we have $\left(N_{T, a+}^{\alpha, b} f\right)(t)=T(b-t, \alpha) f^{\prime}(t)$, similar to the left one. The interested reader can consult [19] to study the properties of operator $N_{T}^{\alpha}$ and additional details.

Similar calculations as in [7] show that the following properties hold.

Theorem 1.4 Let $f$ and $g$ be $N_{T}^{\alpha}$-differentiable at $t>0$ and $\alpha \in(0,1)$. Then
a) $N_{T}^{\alpha}(a f+b g)(t)=a N_{T}^{\alpha}(f)(t)+b N_{T}^{\alpha}(g)(t)$;
b) $N_{T}^{\alpha}\left(t^{p}\right)=T(t, \alpha) p t^{p-1}, p \in \mathbb{R}$;
c) $N_{T}^{\alpha}(\lambda)=0, \lambda \in \mathbb{R}$
d) $N_{T}^{\alpha}(f g)(t)=f N_{T}^{\alpha}(g)(t)+g N_{T}^{\alpha}(f)(t)$;
e) $N_{T}^{\alpha}\left(\frac{f}{g}\right)(t)=\frac{g N_{T}^{\alpha}(f)(t)-f N_{T}^{\alpha}(g)(t)}{g^{2}(t)}$
f) If, in addition, $f$ is differentiable, then $N_{T}^{\alpha}(f)(t)=T(t, \alpha) f^{\prime}(t)$.

Remark 1.5 The relations a), c), d), and e) are similar to the classical results mathematical analysis, and these relationships are not established (or do not occur) for fractional derivatives of global character (see [12] and [20] and the references cited there).

The following statement is analogous to the one known from the ordinary calculus (for a similar result, see [18]).

Theorem 1.6 Letf be an $N_{T}^{\alpha}$-differentiable function on $\left(t_{0}, \infty\right)$. Then, for all $t>t_{0}$, we have

a) Iff is differentiable, then $J_{T, t_{0}}^{\alpha}\left(N_{T}^{\alpha} f(t)\right)=f(t)-f\left(t_{0}\right)$.

b) $N_{T}^{\alpha}\left(J_{T, t_{0}}^{\alpha} f(t)\right)=f(t)$. 
Proof a) By definition, one can calculate

$$
J_{T, t_{0}}^{\alpha}\left(N_{T}^{\alpha} f(t)\right)=\int_{t_{0}}^{t} \frac{N_{T}^{\alpha} f(s)}{T(s, \alpha)} d s=\int_{t_{0}}^{t} \frac{T(s, \alpha) f^{\prime}(s)}{T(s, \alpha)} d s=f(t)-f\left(t_{0}\right) .
$$

b) Analogously, we have

$$
N_{T}^{\alpha}\left(J_{T, t_{0}}^{\alpha} f(t)\right)=T(t, \alpha) \frac{d}{d t}\left[\int_{t_{0}}^{t} \frac{f(s)}{T(s, \alpha)} d s\right]=f(t) .
$$

It is clear that many "classical" properties of integration theory can be proved without much difficulty, for example, the well-known mean value theorems for integral calculus or integration by parts.

Theorem 1.7 Let $f$ and $g$ be differentiable functions on $(a, b), a<b$. Then

$$
J_{T, a}^{\alpha}\left\{\left[N_{T}^{\alpha} f(t)\right] g(t)\right\}(b)=[f(t) g(t)]_{a}^{b}-J_{T, a}^{\alpha}\left\{f(t)\left[N_{T}^{\alpha} g(t)\right]\right\}(b) .
$$

In [5], the so-called Hermite-Hadamard-Fejér inequality was established; it is the weighted generalization of the Hermite-Hadamard inequality (1.1).

Theorem 1.8 Let $f:[a, b] \rightarrow \mathbb{R}$ be a convex function and $g:[a, b] \rightarrow \mathbb{R}_{0}^{+}=[0, \infty)$ be an integrable function symmetric about $\frac{a+b}{2}$. Then

$$
f\left(\frac{a+b}{2}\right) \int_{a}^{b} g(x) d x \leq \int_{a}^{b} f(x) g(x) d x \leq \frac{f(a)+f(b)}{2} \int_{a}^{b} g(x) d x .
$$

In this paper, we present a version of the Hermite-Hadamard-Fejér inequality for convex functions via generalized integral of Definition 1.2, while several remarks presented show the advantage of our framework.

\section{Main results}

Lemma 2.1 Let $g:[a, b] \rightarrow \mathbb{R}, a<b$, be an integrable function symmetric with respect to $\frac{a+b}{2}$. Then we have

$$
J_{T, a+}^{\alpha} g(b)=J_{T, b-}^{\alpha} g(a)=\frac{J_{T, a+}^{\alpha} g(b)+J_{T, b-}^{\alpha} g(a)}{2} .
$$

Proof Following [9] and taking into account the symmetry of $g$ with respect to $\frac{a+b}{2}$, we have

$$
\begin{aligned}
J_{T, a+}^{\alpha} g(b) & =\int_{a}^{b} \frac{g(t)}{T(b-t, \alpha)} d t=\int_{a}^{b} \frac{g(a+b-x)}{T(x-a, \alpha)} d x \\
& =\int_{a}^{b} \frac{g(x)}{T(x-a, \alpha)} d x=J_{T, b-}^{\alpha} g(a) .
\end{aligned}
$$

This immediately implies the result needed.

Remark 2.2 In case of $T(t, \alpha)=t^{1-\alpha} \Gamma(\alpha), \alpha>0$, Lemma 2.1 becomes [9, Lemma 2.1]. 
Theorem 2.3 Let $f:[a, b] \rightarrow \mathbb{R}, a<b$, be a convex function and $g:[a, b] \rightarrow \mathbb{R}_{0}^{+}$be an integrable function symmetric about $\frac{a+b}{2}$. Then

$$
\begin{aligned}
& f\left(\frac{a+b}{2}\right)\left[(a, b) J_{T, a+}^{\alpha}(g)(b)+{ }_{(a, b)} J_{T, b-}^{\alpha}(g)(a)\right] \\
& \quad \leq(a, b) J_{T, a+}^{\alpha}(f g)(b)+(a, b) J_{T, b-}^{\alpha}(f g)(a) \\
& \quad \leq \frac{f(a)+f(b)}{2}\left[(a, b) J_{T, a+}^{\alpha}(g)(b)+{ }_{(a, b)} J_{T, b-}^{\alpha}(g)(a)\right] .
\end{aligned}
$$

Proof From the convexity of $f$, we have

$$
\begin{aligned}
2 f\left(\frac{a+b}{2}\right) & =2 f\left(\frac{t a+(1-t) b+t b+(1-t) a}{2}\right) \\
& \leq f(t a+(1-t) b)+f(t b+(1-t) a)
\end{aligned}
$$

for any $t \in[0,1]$. Multiplying the first and the last members of this inequality by $\frac{g(t b+(1-t) a)}{T(t, \alpha)}$ and integrating on $[0,1]$, we obtain

$$
\begin{aligned}
2 f( & \left.\frac{a+b}{2}\right) \int_{0}^{1} \frac{g(t b+(1-t) a)}{T(t, \alpha)} d t \\
\leq & \int_{0}^{1} \frac{g(t b+(1-t) a)[f(t a+(1-t) b)+f(t b+(1-t) a)]}{T(t, \alpha)} d t \\
= & \int_{0}^{1} \frac{g(t b+(1-t) a) f(t a+(1-t) b)}{T(t, \alpha)} d t \\
& \quad+\int_{0}^{1} \frac{g(t b+(1-t) a) f(t b+(1-t) a)}{T(t, \alpha)} d t .
\end{aligned}
$$

Making the change of variables $x=t b+(1-t) a)$, we obtain

$$
\begin{aligned}
& \frac{2 f\left(\frac{a+b}{2}\right)}{b-a} \int_{a}^{b} \frac{g(x)}{T\left(\frac{x-a}{b-a}, \alpha\right)} d x \\
& \quad \leq \frac{1}{b-a} \int_{a}^{b} \frac{g(x) f(a+b-x)}{T\left(\frac{x-a}{b-a}, \alpha\right)} d x+\frac{1}{b-a} \int_{a}^{b} \frac{g(x) f(x)}{T\left(\frac{x-a}{b-a}, \alpha\right)} d x \\
& \quad=\frac{1}{b-a} \int_{a}^{b} \frac{g(a+b-x) f(x)}{T\left(\frac{b-x}{b-a}, \alpha\right)} d x+\frac{1}{b-a} \int_{a}^{b} \frac{g(x) f(x)}{T\left(\frac{x-a}{b-a}, \alpha\right)} d x \\
& \quad=\frac{1}{b-a} \int_{a}^{b} \frac{g(x) f(x)}{T\left(\frac{b-x}{b-a}, \alpha\right)} d x+\frac{1}{b-a} \int_{a}^{b} \frac{g(x) f(x)}{T\left(\frac{x-a}{b-a}, \alpha\right)} d x .
\end{aligned}
$$

This means

$$
2 f\left(\frac{a+b}{2}\right){ }_{(a, b)} J_{T, a+}^{\alpha}(g)(b) \leq(a, b) J_{T, a+}^{\alpha}(f g)(b)+{ }_{(a, b)} J_{T, b-}^{\alpha}(f g)(a) .
$$

Using Lemma 2.1, we obtain the first inequality in (2.1).

To prove the second part of (2.1), we use the convexity of $f$. We have

$$
f(t a+(1-t) b)+f(t b+(1-t) a) \leq f(a)+f(b)
$$


for any $t \in[0,1]$. Multiplying the previous inequality by $\frac{g(t b+(1-t) a)}{T(t, \alpha)}$ and integrating on $[0,1]$, we obtain, after making the change of variables and considering the symmetry of $g$, the following inequality:

$$
(a, b) J_{T, a+}^{\alpha}(f g)(b)+{ }_{(a, b)} J_{T, b-}^{\alpha}(f g)(a) \leq(f(a)+f(b))_{(a, b)} J_{T, a+}^{\alpha}(g)(b) .
$$

Lemma 2.1 implies the second part of (2.1). This completes the proof of the theorem.

Remark 2.4 If we consider $T(t, \alpha) \equiv 1$, inequality (2.1) becomes the classic HermiteHadamard-Fejér inequality (1.2). In case of $T(t, \alpha)=t^{1-\alpha} \Gamma(\alpha)$, the previous result becomes [9, Theorem 2.2], and if $T(t, \alpha)=t^{1-\alpha} \Gamma(\alpha)$ and $g(x) \equiv 1$, then the above result becomes [25, Theorem 2].

Lemma 2.5 Let $f:[a, b] \rightarrow \mathbb{R}$ be a differentiable function on $(a, b), a<b$, such that $N_{T}^{\alpha} f \in$ $L(a, b)$. If in addition $g:[a, b] \rightarrow \mathbb{R}$ is integrable and symmetric to $\frac{a+b}{2}$, then the following equality is valid:

$$
\begin{gathered}
J_{T, a}^{\alpha}\left\{\left[N_{T}^{\alpha} f(t)\right] \int_{a}^{t} \frac{g(s)}{T(b-s, \alpha)} d s\right\}(b)+J_{T, a}^{\alpha}\left\{-\left[N_{T}^{\alpha} f(t)\right] \int_{t}^{b} \frac{g(s)}{T(s-a, \alpha)} d s\right\}(b) \\
=\frac{f(a)+f(b)}{2}\left[J_{T, a+}^{\alpha}(g)(b)+J_{T, b-}^{\alpha}(g)(a)\right]-\left[J_{T, a+}^{\alpha}(f g)(b)+J_{T, b-}^{\alpha}(f g)(a)\right] .
\end{gathered}
$$

Proof Using Theorem 1.7, we calculate as follows:

$$
\begin{aligned}
J_{1} & =J_{T, a}^{\alpha}\left\{\left[N_{T}^{\alpha} f(t)\right] \int_{a}^{t} \frac{g(s)}{T(b-s, \alpha)} d s\right\}(b) \\
& =\left[f(t) \int_{a}^{t} \frac{g(s)}{T(b-s, \alpha)} d s\right]_{a}^{b}-J_{T, a}^{\alpha}\left\{\frac{f(t) g(t) T(t, \alpha)}{T(b-t, \alpha)}\right\}(b) \\
& =f(b) J_{T, a+}^{\alpha}(g)(b)-J_{T, a+}^{\alpha}(f g)(b)
\end{aligned}
$$

and

$$
\begin{aligned}
J_{2} & =J_{T, a}^{\alpha}\left\{-\left[N_{T}^{\alpha} f(t)\right] \int_{t}^{b} \frac{g(s)}{T(s-a, \alpha)} d s\right\}(b) \\
& =\left[-f(t) \int_{t}^{b} \frac{g(s)}{T(s-a, \alpha)} d s\right]_{a}^{b}-J_{T, a}^{\alpha}\left\{\frac{f(t) g(t) T(t, \alpha)}{T(t-a, \alpha)}\right\}(b) \\
& =f(a) J_{T, b-}^{\alpha}(g)(a)-J_{T, b-}^{\alpha}(f g)(a) .
\end{aligned}
$$

According to Lemma 2.1, we have

$$
\begin{aligned}
& J_{1}=f(b)\left[\frac{J_{T, a+}^{\alpha}(g)(b)+J_{T, b_{-}}^{\alpha}(g)(a)}{2}\right]-J_{T, a+}^{\alpha}(f g)(b), \\
& J_{2}=f(a)\left[\frac{J_{T, a+}^{\alpha}(g)(b)+J_{T, b_{-}}^{\alpha}(g)(a)}{2}\right]-J_{T, b_{-}}^{\alpha}(f g)(a),
\end{aligned}
$$

from where the desired equality is obtained. 
Remark 2.6 In case of $T(t, \alpha)=t^{1-\alpha} \Gamma(\alpha)$, Lemma 2.5 implies [9, Theorem 2.4], and if $T(t, \alpha)=t^{1-\alpha} \Gamma(\alpha)$ and $g(x) \equiv 1$, then Lemma 2.5 implies [25, Lemma 2].

Theorem 2.7 Let $f:[a, b] \rightarrow \mathbb{R}$ be a differentiable function on $(a, b), a<b$, such that $f^{\prime} \in$ $L(a, b),\left|T^{-1}\right| \in L(0, b-a)$. If we also assume that $\left|f^{\prime}\right|$ is convex on $[a, b]$ and $g:[a, b] \rightarrow \mathbb{R}$ is integrable and symmetric to $\frac{a+b}{2}$, then the following inequality holds:

$$
\begin{aligned}
& \left|\frac{f(a)+f(b)}{2}\left[J_{T, a+}^{\alpha}(g)(b)+J_{T, b-}^{\alpha}(g)(a)\right]-\left[J_{T, a+}^{\alpha}(f g)(b)+J_{T, b-}^{\alpha}(f g)(a)\right]\right| \\
& \quad \leq\|g\|_{\infty}\left(\left|f^{\prime}(a)\right|+\left|f^{\prime}(b)\right|\right)\left[\int_{\frac{b-a}{2}}^{b-a} \mathcal{T}(s) d s-\int_{0}^{\frac{b-a}{2}} \mathcal{T}(s) d s\right],
\end{aligned}
$$

where $\mathcal{T}(s)=\int_{0}^{s} \frac{d t}{|T(t, \alpha)|}$.

Proof Let us see some preliminary details. First, note that (2.2) is equivalent to

$$
\begin{aligned}
& \int_{a}^{b}\left\{\int_{a}^{t} \frac{g(s)}{T(b-s, \alpha)} d s-\int_{t}^{b} \frac{g(s)}{T(s-a, \alpha)} d s\right\} f^{\prime}(t) d t \\
& \quad=\frac{f(a)+f(b)}{2}\left[J_{T, a+}^{\alpha}(g)(b)+J_{T, b-}^{\alpha}(g)(a)\right]-\left[J_{T, a+}^{\alpha}(f g)(b)+J_{T, b-}^{\alpha}(f g)(a)\right] .
\end{aligned}
$$

Since $\left|f^{\prime}\right|$ is convex, we have

$$
\left|f^{\prime}(t)\right|=\left|f^{\prime}\left(\frac{b-t}{b-a} a+\frac{t-a}{b-a} b\right)\right| \leq \frac{1}{b-a}\left((b-t)\left|f^{\prime}(a)\right|+(t-a)\left|f^{\prime}(b)\right|\right)
$$

for any $t \in[a, b]$. Using (2.3) and the symmetry of $g$, we get

$$
\begin{aligned}
&\left|\frac{f(a)+f(b)}{2}\left[J_{T, a+}^{\alpha}(g)(b)+J_{T, b-}^{\alpha}(g)(a)\right]-\left[J_{T, a+}^{\alpha}(f g)(b)+J_{T, b-}^{\alpha}(f g)(a)\right]\right| \int_{a}^{b}\left|\int_{a}^{t} \frac{g(s)}{T(b-s, \alpha)} d s-\int_{t}^{b} \frac{g(s)}{T(s-a, \alpha)} d s\right|\left|f^{\prime}(t)\right| d t \\
& \quad=\int_{a}^{b}\left|\int_{a}^{t} \frac{g(s)}{T(b-s, \alpha)} d s-\int_{a}^{a+b-t} \frac{g(s)}{T(b-s, \alpha)} d s\right| f^{\prime}(t) \mid d t \\
&=\int_{a}^{b}\left|f^{\prime}(t)\right|\left|\int_{t}^{a+b-t} \frac{g(s)}{T(b-s, \alpha)} d s\right| d t \\
& \leq\|g\|_{\infty} \int_{a}^{\frac{a+b}{2}}\left|f^{\prime}(t)\right|\left\{\int_{t}^{a+b-t} \frac{d s}{|T(b-s, \alpha)|}\right\} d t \\
& \quad+\|g\|_{\infty} \int_{\frac{a+b}{2}}^{b}\left|f^{\prime}(t)\right|\left\{\int_{a+b-t}^{t} \frac{d s}{|T(b-s, \alpha)|}\right\} d t \\
& \quad+\|g\|_{\infty} \int_{\frac{a+b}{2}}^{b}\left|f^{\prime}(t)\right|\left\{\int_{b-t}^{t-a} \frac{d s}{|T(s, \alpha)|}\right\} d t, \\
& \quad \| g \int_{a}^{\frac{a+b}{2}}\left|f^{\prime}(t)\right|\left\{\int_{t-a}^{b-t} \frac{d s}{|T(s, \alpha)|}\right\} d t \\
& \quad
\end{aligned}
$$


from where (2.4) implies

$$
\begin{aligned}
\mid \frac{f(a)}{2}+f(b) & \left.J_{T, a+}^{\alpha}(g)(b)+J_{T, b-}^{\alpha}(g)(a)\right]-\left[J_{T, a+}^{\alpha}(f g)(b)+J_{T, b-}^{\alpha}(f g)(a)\right] \mid \\
\leq & \frac{\|g\|_{\infty}}{b-a} \int_{a}^{\frac{a+b}{2}}\left((b-t)\left|f^{\prime}(a)\right|+(t-a)\left|f^{\prime}(b)\right|\right)(\mathcal{T}(b-t)-\mathcal{T}(t-a)) d t \\
& +\frac{\|g\|_{\infty}}{b-a} \int_{\frac{a+b}{2}}^{b}\left((b-t)\left|f^{\prime}(a)\right|+(t-a)\left|f^{\prime}(b)\right|\right)(\mathcal{T}(t-a)-\mathcal{T}(b-t)) d t \\
= & \frac{\|g\|_{\infty}}{b-a}\left|f^{\prime}(a)\right|\left[\int_{a}^{\frac{a+b}{2}}(b-t) \mathcal{T}(b-t) d t+\int_{\frac{a+b}{2}}^{b}(b-t) \mathcal{T}(t-a) d t\right] \\
& -\frac{\|g\|_{\infty}}{b-a}\left|f^{\prime}(a)\right|\left[\int_{a}^{\frac{a+b}{2}}(b-t) \mathcal{T}(t-a) d t+\int_{\frac{a+b}{2}}^{b}(b-t) \mathcal{T}(b-t) d t\right] \\
& +\frac{\|g\|_{\infty}}{b-a}\left|f^{\prime}(b)\right|\left[\int_{a}^{\frac{a+b}{2}}(t-a) \mathcal{T}(b-t) d t+\int_{\frac{a+b}{2}}^{b}(t-a) \mathcal{T}(t-a) d t\right] \\
& -\frac{\|g\|_{\infty}}{b-a}\left|f^{\prime}(b)\right|\left[\int_{a}^{\frac{a+b}{2}}(t-a) \mathcal{T}(t-a) d t+\int_{\frac{a+b}{2}}^{b}(t-a) \mathcal{T}(b-t) d t\right] \\
= & \frac{\|g\|_{\infty}}{b-a}\left|f^{\prime}(a)\right|\left[\int_{\frac{b-a}{2}}^{b-a} s \mathcal{T}(s) d s+\int_{\frac{b-a}{2}}^{b-a}(b-a-s) \mathcal{T}(s) d s\right] \\
& -\frac{\|g\|_{\infty}}{b-a}\left|f^{\prime}(a)\right|\left[\int_{0}^{\frac{b-a}{2}}(b-a-s) \mathcal{T}(s) d s+\int_{0}^{\frac{b-a}{2}} s \mathcal{T}(s) d s\right] \\
& +\frac{\|g\|_{\infty}}{b-a}\left|f^{\prime}(b)\right|\left[\int_{\frac{b-a}{2}}^{b-a}(b-a-s) \mathcal{T}(s) d s+\int_{\frac{b-a}{2}}^{b-a} s \mathcal{T}(s) d s\right] \\
& -\frac{\|g\|_{\infty}}{b-a}\left|f^{\prime}(b)\right|\left[\int_{0}^{\frac{b-a}{2}} s \mathcal{T}(s) d s+\int_{0}^{\frac{b-a}{2}}(b-a-s) \mathcal{T}(s) d s\right] .
\end{aligned}
$$

Adding up the members on the right-hand side of the last inequality immediately gives the desired inequality.

Remark 2.8 In case of $T(t, \alpha)=t^{1-\alpha} \Gamma(\alpha), \alpha>0$, Theorem 2.7 implies [9, Theorem 2.4].

In a similar way the following result can be proved.

Theorem 2.9 Let $f:[a, b] \rightarrow \mathbb{R}$ be a differentiable function on $(a, b), a<b$, such that $\left|f^{\prime}\right|^{q} \in$ $L(a, b), q>1,\left|T^{-1}\right| \in L(0, b-a)$. If we also assume that $\left|f^{\prime}\right|^{q}$ is convex on $[a, b]$ and $g$ : $[a, b] \rightarrow \mathbb{R}$ is integrable and symmetric to $\frac{a+b}{2}$, then

$$
\begin{aligned}
& \left|\frac{f(a)+f(b)}{2}\left[J_{T, a+}^{\alpha}(g)(b)+J_{T, b-}^{\alpha}(g)(a)\right]-\left[J_{T, a+}^{\alpha}(f g)(b)+J_{T, b-}^{\alpha}(f g)(a)\right]\right| \\
& \quad \leq 2^{1-1 / q}\|g\|_{\infty}\left(\left|f^{\prime}(a)\right|^{q}+\left|f^{\prime}(b)\right|^{q}\right)^{1 / q}\left[\int_{\frac{b-a}{2}}^{b-a} \mathcal{T}(s) d s-\int_{0}^{\frac{b-a}{2}} \mathcal{T}(s) d s\right],
\end{aligned}
$$

where $\mathcal{T}(s)=\int_{0}^{s} \frac{d t}{|T(t, \alpha)|}$. 
Proof Using (2.3) and Hölder's inequality, we get

$$
\begin{aligned}
& \left|\frac{f(a)+f(b)}{2}\left[J_{T, a+}^{\alpha}(g)(b)+J_{T, b-}^{\alpha}(g)(a)\right]-\left[J_{T, a+}^{\alpha}(f g)(b)+J_{T, b-}^{\alpha}(f g)(a)\right]\right| \\
& \quad \leq\left[\int_{a}^{b}\left|\int_{t}^{a+b-t} \frac{g(s)}{T(b-s, \alpha)} d s\right| d t\right]^{1-1 / q} \cdot\left[\int_{a}^{b}\left|f^{\prime}(t)\right|^{q}\left|\int_{t}^{a+b-t} \frac{g(s)}{T(b-s, \alpha)} d s\right| d t\right]^{1 / q} \\
& \quad=I_{1} \cdot I_{2} .
\end{aligned}
$$

Analogously as in the proof of Theorem 2.7, we obtain

$$
I_{2} \leq\|g\|_{\infty}^{1 / q}\left(\left|f^{\prime}(a)\right|^{q}+\left|f^{\prime}(b)\right|^{q}\right)^{1 / q}\left[\int_{\frac{b-a}{2}}^{b-a} \mathcal{T}(s) d s-\int_{0}^{\frac{b-a}{2}} \mathcal{T}(s) d s\right]^{1 / q}
$$

By similar calculation,

$$
\begin{aligned}
I_{1} & \leq\|g\|_{\infty}^{1-1 / q}\left[\int_{a}^{\frac{a+b}{2}}\left\{\int_{t-a}^{b-t} \frac{d s}{|T(s, \alpha)|}\right\} d t+\int_{\frac{a+b}{2}}^{b}\left\{\int_{b-t}^{t-a} \frac{d s}{|T(s, \alpha)|}\right\} d t\right]^{1-1 / q} \\
& =\|g\|_{\infty}^{1-1 / q}\left[\int_{a}^{\frac{a+b}{2}} \mathcal{T}(b-t)-\mathcal{T}(t-a) d t+\int_{\frac{a+b}{2}}^{b} \mathcal{T}(t-a)-\mathcal{T}(b-t) d t\right]^{1-1 / q} \\
& =\|g\|_{\infty}^{1-1 / q}\left[\int_{\frac{b-a}{2}}^{b-a} \mathcal{T}(s) d s-\int_{0}^{\frac{b-a}{2}} \mathcal{T}(s) d s+\int_{\frac{b-a}{2}}^{b-a} \mathcal{T}(s) d s-\int_{0}^{\frac{b-a}{2}} \mathcal{T}(s) d s\right]^{1-1 / q},
\end{aligned}
$$

from where a multiplication gives the desired inequality.

Remark 2.10 In case of $T(t, \alpha)=t^{1-\alpha} \Gamma(\alpha), \alpha>0$, under the conditions of Theorem 2.7, we have

$$
\begin{aligned}
& \left|\frac{f(a)+f(b)}{2}\left[{ }^{R L} J_{a^{+}}^{\alpha}(g)(b)+{ }^{R L} J_{b^{-}}^{\alpha}(g)(a)\right]-\left[{ }^{R L} J_{a^{+}}^{\alpha}(f g)(b)+{ }^{R L} J_{b^{-}}^{\alpha}(f g)(a)\right]\right| \\
& \quad \leq \frac{2(b-a)^{\alpha+1}\|g\|_{\infty}}{\Gamma(\alpha+2)}\left(1-\frac{1}{2^{\alpha}}\right)\left(\frac{\left|f^{\prime}(a)\right|^{q}+\left|f^{\prime}(b)\right|^{q}}{2}\right)^{1 / q}
\end{aligned}
$$

that corrects the result of [9, Theorem 2.8].

Remark 2.11 If we consider $q>1$ and $\frac{1}{p}+\frac{1}{q}=1$, we can obtain other inequalities.

\section{Conclusions}

In this article we study new inequalities of the Hermite-Hadamard-Fejér type for functions with derivatives of special type via convexity. The results obtained allow to extend known results in the literature with an appropriate choice of the kernel, since we use general fractional integrals. For example, let us take $T(t, \alpha)=k \Gamma_{k}(\alpha) t^{1-\alpha / k}, \alpha>0$, and therefore consider the Riemann-Liouville $k$-fractional integrals of order $\alpha>0$ defined by (see $[1,17])$

$$
{ }^{R L} J_{a^{+}}^{\alpha, k} f(t)=\frac{1}{k \Gamma_{k}(\alpha)} \int_{a}^{t}(t-s)^{\alpha / k-1} f(s) d s, \quad t>a,
$$




$$
{ }^{R L} J_{b^{-}}^{\alpha, k} f(t)=\frac{1}{k \Gamma_{k}(\alpha)} \int_{t}^{b}(s-t)^{\alpha / k-1} f(s) d s, \quad t<b .
$$

Under the conditions of Theorem 2.7, we have

$$
\begin{aligned}
& \left|\frac{f(a)+f(b)}{2}\left[{ }^{R L} J_{a^{+}}^{\alpha, k}(g)(b)+{ }^{R L} J_{b^{-}}^{\alpha, k}(g)(a)\right]-\left[{ }^{R L} J_{a^{+}}^{\alpha, k}(f g)(b)+{ }^{R L} J_{b^{-}}^{\alpha, k}(f g)(a)\right]\right| \\
& \quad \leq \frac{(b-a)^{\alpha / k+1}\|g\|_{\infty}}{\Gamma_{k}(\alpha+k)\left(\frac{\alpha}{k}+1\right)}\left(1-\frac{1}{2^{\alpha / k}}\right)\left(\left|f^{\prime}(a)\right|+\left|f^{\prime}(b)\right|\right),
\end{aligned}
$$

while under the conditions of Theorem 2.9,

$$
\begin{aligned}
& \left|\frac{f(a)+f(b)}{2}\left[{ }^{R L} J_{a^{+}}^{\alpha, k}(g)(b)+{ }^{R L} J_{b^{-}}^{\alpha, k}(g)(a)\right]-\left[{ }^{R L} J_{a^{+}}^{\alpha, k}(f g)(b)+{ }^{R L} J_{b^{-}}^{\alpha, k}(f g)(a)\right]\right| \\
& \quad \leq \frac{2(b-a))^{\alpha / k+1}\|g\|_{\infty}}{\Gamma_{k}(\alpha+k)\left(\frac{\alpha}{k}+1\right)}\left(1-\frac{1}{2^{\alpha / k}}\right)\left(\frac{\left|f^{\prime}(a)\right|^{q}+\left|f^{\prime}(b)\right|^{q}}{2}\right)^{1 / q} .
\end{aligned}
$$

The results obtained also open the door to the generalization study with other types of integral inequalities such as Ostrowski, Grüss, Jensen among others, as well as for functions with other types of generalized convexity such as strongly convex, $m$-convex, $s$-convex, relative convex among others. Finally, our results can also be extended to functions of various variables using convexity in coordinates.

\section{Acknowledgements}

We express our special thanks to the editor and referees of this manuscript.

\section{Funding}

Not applicable.

Availability of data and materials

Not applicable.

\section{Competing interests}

The authors declare that they have no competing interests.

\section{Authors' contributions}

The three authors have contributed equally to the attempt. All three authors have read carefully and approved the final version of the study.

\section{Author details}

${ }^{1}$ Faculty of Exact and Natural Sciences, School of Physical Sciences and Mathematics, Pontifical Catholic University of Ecuador, Av. 12 de octubre 1076 y Roca, Apartado Postal 17-01-2184, Sede Quito, Ecuador. ${ }^{2}$ Institute of Applied Pedagogy, Juhász Gyula Faculty of Education, University of Szeged, Hattyas utca 10, H-6725 Szeged, Hungary. ${ }^{3}$ FaCENA, UNNE, Av. Libertad 5450, Corrientes 3400, Argentina. ${ }^{4}$ UTN-FRRE, French 414, Resistencia, Chaco 3500, Argentina.

\section{Publisher's Note}

Springer Nature remains neutral with regard to jurisdictional claims in published maps and institutional affiliations.

Received: 7 January 2021 Accepted: 22 March 2021 Published online: 08 April 2021

\section{References}

1. Agarwal, P., Tariboon, J., Ntouyas, S.K.: Some generalized Riemann-Liouville $k$-fractional integral inequalities. J. Inequal. Appl. 2016, $122(2016)$

2. Baleanu, D., Mohammed, P.O., Zeng, S.: Inequalities of trapezoidal type involving generalized fractional integrals. Alex. Eng. J. 59(5), 2975-2984 (2020)

3. Bessenyei, M., Páles, Z:: On generalized higher-order convexity and Hermite-Hadamard-type inequalities. Acta Sci. Math. 70(1-2), 13-24 (2004)

4. Dragomir, S.S., Pearce, C.E.M.: Selected Topics on Hermite-Hadamard Inequalities and Applications. RGMIA Monographs. Victoria University, Melbourne (2000) 
5. Fejér, L.: Uber die Fourierreihen, II. Math. Naturwiss. Anz. Ungar. Akad. Wiss. 24, 369-390 (1906) (in Hungarian)

6. Gorenflo, R., Mainardi, F.: Fractals and Fractional Calculus in Continuum Mechanics, pp. 223-276. Springer, Wien (1997)

7. Guzmán, P.M., Langton, G., Lugo, L.M., Medina, J., Nápoles Valdés, J.E.: A new definition of a fractional derivative of local type. J. Math. Anal. 9(2), 88-98 (2018)

8. Guzmán, P.M., Lugo, L.M., Nápoles Valdés, J.E., Vivas-Cortez, M.: On a new generalized integral operator and certain operating properties. Axioms 9(2), 69 (2020)

9. Iş̧can, i.: Hermite-Hadamard-Fejér type inequalities for convex functions via fractional integrals. Stud. Univ. Babeş-Bolyai, Math. 60(3), 355-366 (2015)

10. Jain, S., Mehrez, K., Baleanu, D., Agarwal, P.: Certain Hermite-Hadamard Inequalities for Logarithmically Convex Functions with Applications. Mathematics 7(2), 163 (2019)

11. Jarad, F., Uğurlu, E., Abdeljawad, T., Baleanu, D.: On a new class of fractional operators. Adv. Differ. Equ. 2017, 247 (2017)

12. Kilbas, A., Srivastava, M.H., Trujillo, J.J.: Theory and Application on Fractional Differential Equations. North-Holland, Amsterdam (2006)

13. Klaričić, M., Neuman, E., Pečarić, J., Šimić, V.: Hermite-Hadamard's inequalities for multivariate $g$-convex functions. Math. Inequal. Appl. 8(2), 305-316 (2005)

14. Kórus, P., Lugo, L.M., Nápoles Valdés, J.E.: Integral inequalities in a generalized context. Studia Sci. Math. Hung. 57, 312-320 (2020)

15. Mohammed, P.O., Sarikaya, M.Z., Baleanu, D.: On the generalized Hermite-Hadamard inequalities via the tempered fractional integrals. Symmetry 12(4), 595 (2020)

16. Moslehian, M.S.: Matrix Hermite-Hadamard type inequalities. Houst. J. Math. 39(1), 177-189 (2013)

17. Mubeen, S., Habibullah, G.M.: k-Fractional integrals and application. Int. J. Contemp. Math. Sci. 7, 89-94 (2012)

18. Nápoles Valdés, J.E., Guzmán, P.M., Lugo, L.M.: Some new results on nonconformable fractional calculus. Adv. Dyn. Syst. Appl. 13(2), 167-175 (2018)

19. Nápoles Valdés, J.E., Guzmán, P.M., Lugo, L.M., Kashuri, A.: The local non-conformable derivative and Mittag-Leffler function. Sigma J. Eng. Nat. Sci. 38(2), 1007-1017 (2020)

20. Podlubny, I.: Fractional Differential Equations. Academic Press, San Diego (1999)

21. Rangel-Oliveros, Y.C., Vivas-Cortez, M.J.: On some Hermite-Hadamard type inequalities for functions whose second derivatives are convex generalized. Revista MATUA 5(2), 21-31 (2018)

22. Rangel-Oliveros, Y.C., Vivas-Cortez, M.J.: Ostrowski type inequalities for functions whose second derivative are convex generalized. Appl. Math. Inf. Sci. 12(6), 1117-1126 (2018)

23. Rashid, S., İscan, I.., Baleanu, D., Chu, Y.-M.: Generation of new fractional inequalities via $n$ polynomials s-type convexity with applications. Adv. Differ. Equ. 2020, 264 (2020)

24. Saoudi, K., Agarwal, P., Kumam, P., Ghanmi, A., Thounthonget, P.: The Nehari manifold for a boundary value problem involving Riemann-Liouville fractional derivative. Adv. Differ. Equ. 2018, 263 (2018)

25. Sarikaya, M.Z., Set, E., Yaldiz, H., Başak, N.: Hermite-Hadamard's inequalities for fractional integrals and related fractional inequalities. Math. Comput. Model. 57, 2403-2407 (2013)

26. Sitho, S., Ntouyas, S.K., Agarwal, P., Tariboon, J.: Noninstantaneous impulsive inequalities via conformable fractional calculus. J. Inequal. Appl. 2018, 261 (2018)

27. Tomar, M., Agarwal, P., Choi, J.: Hermite-Hadamard type inequalities for generalized convex functions on fractal sets style. Bol. Soc. Parana. Mat. 38(1), 101-116 (2020)

28. Vivas-Cortez, M.: Féjer type inequalities for ( $(,, m)$-convex functions in the second sense. Appl. Math. Inf. Sci. 10(5), 1689-1696 (2016)

29. Vivas-Cortez, M., Abdeljawad, T., Mohammed, P.O., Rangel-Oliveros, Y.: Simpson's integral inequalities for twice differentiable convex functions. Math. Probl. Eng. 2020, Article ID 1936461 (2020)

30. Vivas-Cortez, M., Rangel-Oliveros, Y.: An inequality related to $s-\varphi$-convex functions. Appl. Math. Inf. Sci. 14, 151-154 (2020)

31. Zhao, D., Luo, M.: General conformable fractional derivative and its physical interpretation. Calcolo 54, 903-917 (2017)

\section{Submit your manuscript to a SpringerOpen ${ }^{\circ}$ journal and benefit from:}

- Convenient online submission

- Rigorous peer review

- Open access: articles freely available online

- High visibility within the field

- Retaining the copyright to your article

Submit your next manuscript at $\gg$ springeropen.com 